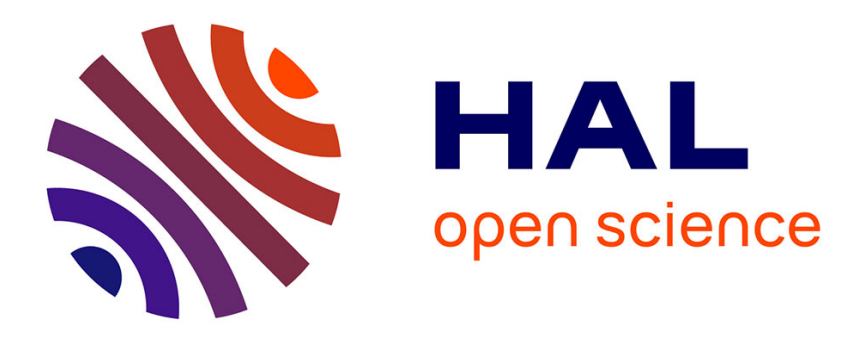

\title{
Etude et mise en œuvre d'une chaîne d'acquisition de mesures basée sur l'utilisation de magnétophones à cassette
}

\author{
V. Mercier
}

\section{- To cite this version:}

V. Mercier. Etude et mise en œuvre d'une chaîne d'acquisition de mesures basée sur l'utilisation de magnétophones à cassette. Revue de Physique Appliquée, 1986, 21 (1), pp.59-63. 10.1051/rphysap:0198600210105900 . jpa-00245411

HAL Id: jpa-00245411

https://hal.science/jpa-00245411

Submitted on 1 Jan 1986

HAL is a multi-disciplinary open access archive for the deposit and dissemination of scientific research documents, whether they are published or not. The documents may come from teaching and research institutions in France or abroad, or from public or private research centers.
L'archive ouverte pluridisciplinaire HAL, est destinée au dépôt et à la diffusion de documents scientifiques de niveau recherche, publiés ou non, émanant des établissements d'enseignement et de recherche français ou étrangers, des laboratoires publics ou privés. 
Classification

Physics Abstracts

$06.70-07.50-47.80$

\title{
Etude et mise en œuvre d'une chaîne d'acquisition de mesures basée sur l'utilisation de magnétophones à cassette
}

\author{
V. Mercier \\ Laboratoire d'Etudes Aérodynamiques, (CNRS LA 191), Centre d'Etudes Aérodynamiques et Thermiques, \\ 43, rue de l'Aérodrome, 86000 Poitiers, France
}

(Reçu le 21 décembre 1984, révisé les 27 juin et 17 septembre 1985, accepté le 23 septembre 1985)

\begin{abstract}
Résumé. - Nous avons étudié et mis en œuvre une chaîne d'acquisition de mesure de phénomènes variables dans le temps. De fabrication et d'emploi économiques, elle est utilisable sur banc d'essai en laboratoire ou sur site, le traitement différé se faisant ensuite par ordinateur. Elle est constituée d'interfaces originales d'enregistrement et de lecture conçues en association avec des magnétophones stéréophoniques à cassette de type " grand public ». Le signal, analogique ou logique, est enregistré sur l'une des voies tandis qu'un signal d'horloge, enregistré sur l'autre, rend possible la synchronisation de plusieurs mesures. L'ensemble permet l'exploitation de tensions comprises entre $50 \mathrm{mV}$ et $10 \mathrm{~V}$ et de fréquences allant de 8 à $400 \mathrm{~Hz}$ en régime permanent avec une précision meilleure que $0,8 \%$ et en régime transitoire, des variations de tension d'entrée de $4 \mathrm{~V}$ par $1 / 10^{\mathrm{e}}$ de seconde et de fréquence jusqu'à $80 \mathrm{~Hz}$ par $1 / 10^{\mathrm{e}}$ de seconde. La consommation par voie n'excède pas $130 \mathrm{~mA}$. Un exemple d'acquisition des mesures réalisées sur une éolienne placée sur site est présenté.
\end{abstract}

\begin{abstract}
We have studied and implemented a data acquisition system for variable phenomena. It is of cheap price, easy to use and can be employed in Laboratory or in situ experimental set up. The signal delayed processing is then carried out by means of a computer. It includes tape recording and playing back original interfaces conceived for use with no professional stereophonic cassette recorders. The analog or logic signal is recorded on a track while a clock signal, recorded on the other track, permits synchronization of several measures. The system makes possible the processing of voltage signals from $50 \mathrm{mV}$ to $10 \mathrm{~V}$ and signal frequencies from $8 \mathrm{~Hz}$ to $400 \mathrm{~Hz}$ on continuous working conditions with a precision better than $0.8 \%$ and on transient working conditions the processing of input signals voltages varying of $4 \mathrm{~V}$ per 0.1 second and variation frequency of $80 \mathrm{~Hz}$ per 0.1 second. The consumption per channel does not exceed $130 \mathrm{~mA}$. An example of in situ wind turbine data acquisition is shown here.
\end{abstract}

\section{Introduction.}

Il est courant d'avoir à étudier expérimentalement l'évolution de phénomènes modérément variables sur des temps assez longs. Lorsqu'en plus, le traitement des mesures ne peut s'effectuer en temps réel (éloignement de l'ordinateur ou durée de traitement prohibitive pour les autres utilisateurs en temps partagé), il est intéressant d'acquérir les informations et de les stocker sur une mémoire intermédiaire en vue d'un traitement ultérieur par ordinateur. L'utilisation d'un enregistreur magnétique continu s'impose dans la plupart des cas, vu la quantité d'informations à traiter, mais ce type d'appareils est onéreux. Le procédé qui est décrit ici apporte une solution économique à ce problème car il est basé sur l'utilisation de magnétophones stéréophoniques à cassettes « grand public ». Ce matériel facilement transportable et de faible consommation, est idéal pour l'équipement de bancs d'essais à fonctionnement intermittent et les mesures effectuées sur site.

Après un bref exposé au paragraphe 2 du principe du procédé d'acquisition de données développé au C.E.A.T., les interfaces sont décrites au paragraphe 3 et les performances de l'ensemble au paragraphe 4 . Le matériel ayant été mis au point dans le cadre d'une étude de fonctionnement d'éolienne entreprise en collaboration avec le Service Technique des Phares et Balises, il a pu être essayé sur le site de Vitrolles et quelques résultats sont présentés à titre d'exemple d'utilisation au paragraphe 5 .

\section{Principe.}

La fréquence $F$ d'un signal enregistré est d'autant mieux restituée à la lecture que les taux de pleurage 
et de scintillement de l'appareil sont faibles et que les vitesses d'enregistrement et de lecture sont égales ou très voisines (un facteur correctif peut être apporté dans ce cas). La bande passante à - $6 \mathrm{~dB}$ de l'appareil définit la gamme de fréquences acceptables. Donc le signal à enregistrer, analogique ou logique, doit être traité afin d'être adapté aux performances du magnétophone.

De même, à la relecture, il devra être adapté au traitement sur ordinateur.

2.1 EnRegistrement. - Deux types d'interface sont donc étudiés selon la nature du signal :

- Le signal analogique est tout d'abord transformé (s'il ne l'est déjà) en tension représentant la grandeur mesurée. On réalise ensuite une conversion tensionfréquence qui fournit un signal en créneaux, de fréquence $F$ proportionnelle à la tension. Cette information est enregistrée sur la première piste du magnétophone stéréophonique. Le facteur d'échelle du convertisseur est choisi en fonction de la plage de variation maximale de la mesure et de la bande passante du magnétophone (à $-6 \mathrm{~dB}$ ).

- Le signal logique (celui issu par exemple d'un détecteur optique de comptage) nécessite une adaptation avant d'être enregistré car sa fréquence est trop basse. Elle doit être multipliée par un facteur constant afin d'entrer dans la bande passante de l'enregistreur. Cette opération est réalisée à l'aide d'une boucle à verrouillage de phase. Chaque voie de mesure est ainsi équipée d'un magnétophone et d'une interface d'enregistrement correspondant à son type. Ceci nécessite une synchronisation des voies entre elles à partir d'un signal d'horloge enregistré sur la deuxième piste des magnétophones.

2.2 LeCTURE. - Sur chaque cassette sont enregistrés le signal d'horloge et un signal de fréquence $F$ représentant la mesure. L'interface de dépouillement basée sur l'utilisation d'un convertisseur fréquencetension fournit une tension proportionnelle à la fréquence enregistrée. Sous cette forme, le signal est alors susceptible d'être traité d'une manière classique sur ordinateur.

Après remise en forme et traitement des signaux, le signal d'horloge est utilisé pour synchroniser le démarrage des acquisitions sur chaque cassette.

\section{Description des interfaces.}

3.1 INTERFACE D'ENREGistrement. - Le schéma synoptique du traitement d'un signal analogique est représenté sur la figure 1 .

La forte impédance d'entrée de l'adaptateur permet l'exploitation en tension de signaux issus de capteurs les plus divers. Le convertisseur tension-fréquence utilisé est le modèle AD 537 d'Analog Devices (Guide d'applications des convertisseurs tension-fréquence AD 537). Sa fonction de transfert est de la forme

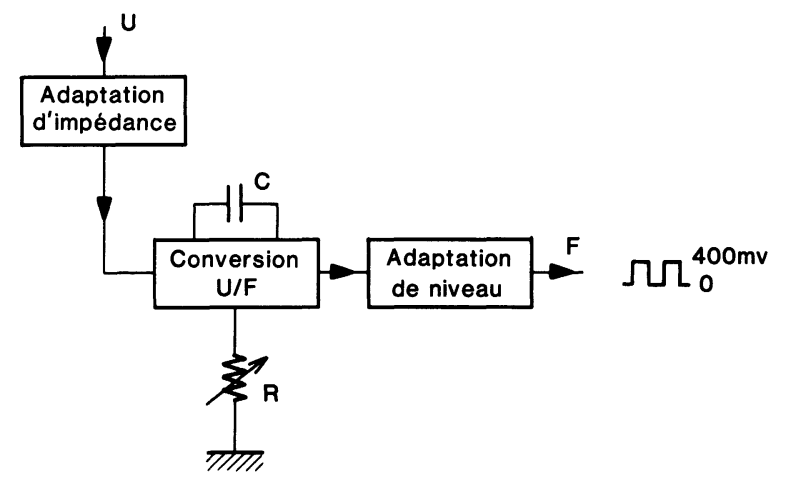

Fig. 1. - Schéma synoptique du traitement d'un signal analogique avant enregistrement.

[Block diagram of the processing of analog signal before tape recording.]

$F=\frac{U}{10 R C}$. Il est aussi utilisable en convertisseur fréquence-tension. Les critères qui conduisent à ce choix sont :

- son faible coût

- sa faible consommation

- sa grande souplesse d'utilisation [1].

Le facteur d'échelle est de $10 \mathrm{kHz}$ pour 10 volts.

Pour les bas niveaux, les performances sont limitées par celles du magnétophone. En effet, pour un signal inférieur à $50 \mathrm{mV}$, la fréquence délivrée est en dehors de la bande passante à $-6 \mathrm{~dB}$ de l'enregistreur. Dans de nombreux cas de mesure, on peut pallier cet inconvénient en procédant à un décalage d'échelle.

Le signal délivré par le module de conversion est ensuite adapté en niveau à l'entrée du magnétophone.

- Le schéma synoptique du traitement d'un signal logique est représenté sur la figure 2 .

Le multiplicateur de fréquences est destiné à traiter des signaux en créneaux présentant un rapport cyclique de 1. Dans la plupart des cas, il est donc nécessaire de procéder à une mise en forme des informations issues de capteurs.

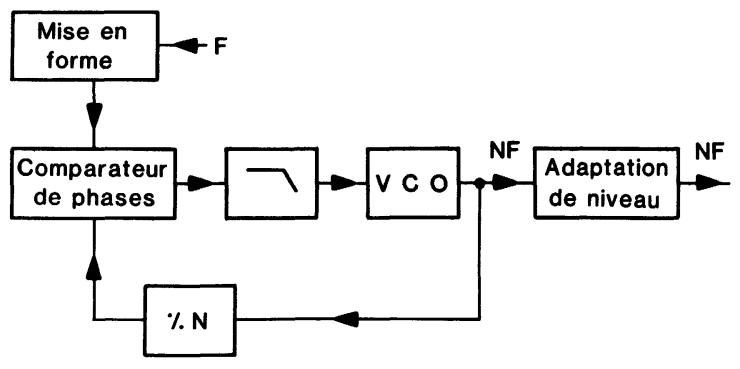

Fig. 2. - Schéma synoptique du traitement d'un signal logique avant enregistrement.

[Processing block diagram of logic signal before tape recording.] 
La multiplication de fréquences se fait au moyen d'une boucle à verrouillage de phase dont les plages de capture et de verrouillage sont ajustées afin de répondre à une étendue de fréquences allant de $8 \mathrm{~Hz}$ à $400 \mathrm{~Hz}$. Le filtre de boucle est un filtre passe-bas passif avec correcteur par retard de phase [2], [3].

Ses composants sont calculés de manière à ce que soient obtenues en même temps, une bonne stabilité en fréquences du VCO de la boucle et une réponse assez rapide de la boucle aux variations brusques de la fréquence incidente. Mais ceci agit sur la plage de capture de la boucle [4]. Il a donc fallu trouver un compromis permettant d'assurer à la fois une bonne stabilité et une réponse assez rapide pour une large gamme de fréquences d'entrée. La réponse de cette boucle est une fonction du second ordre.

Comme dans le cas précédent, il est nécessaire d'adapter en niveau le signal issu du multiplicateur avant son entrée sur le magnétophone.

3.2 Signal D'HORLOGE. - Un multivibrateur astable en technologie CMOS délivre un signal de fréquence $10 \mathrm{~Hz}$ (dont les variations relatives sont inférieures à $0,1 \%$ ).

Ce signal est ensuite traité de façon à fournir des trains d'impulsions de durée $\Delta t_{1}=160 \mathrm{~s}$. L'intervalle de temps $\Delta t_{2}$ séparant deux trains successifs est de $1 \mathrm{~s}$. La procédure d'enregistrement est alors la suivante : démarrage des enregistrements puis lancement du signal d'horloge commun à toutes les voies, les pistes horloge étant mises en parallèle. La resynchronisation des voies entre elles se fait lors de l'exploitation par ordinateur.

$\mathrm{Au}$ traitement, l'information présente sur la piste horloge est mise en forme de manière à obtenir un changement de niveau du signal pour chaque « blanc ». La lecture de chaque cassette est lancée automatiquement par programme en utilisant la fonction télécommande de l'enregistreur et on procède alors à un échantillonnage sur les voies horloge et mesure. Les informations présentes sur la voie mesure sont mémorisées sous forme de "blocs" de durée légèrement inférieure à $160 \mathrm{~s}$ (temps de stockage compatible avec la capacité mémoire de l'ordinateur) dont le début est reconnu par le changement de niveau sur la piste horloge.

C'est ainsi que sont resynchronisées les cassettes entre elles.

3.3 INTERFACE DE LECTURE. - Les informations mémorisées sur les cassettes sont :

- sur une piste, les données de mesures

- sur l'autre piste, les signaux de synchronisation.

- Les données de mesures sont traitées suivant le schéma synoptique de la figure 3 .

Ce signal appliqué à un comparateur rapide est mis en forme avant d'attaquer un module de conversion fréquence-tension, réalisé en utilisant un convertisseur du même type que celui de l'interface d'enre-

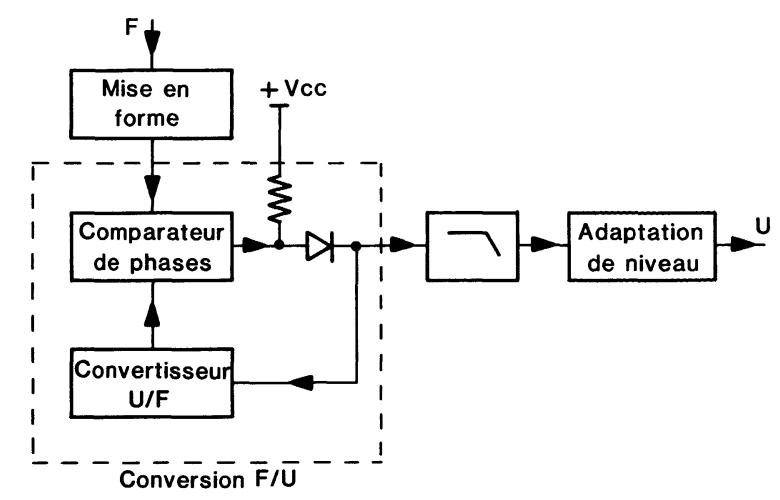

Fig. 3. - Schéma synoptique du traitement des données de mesures enregistrées.

[Block diagram of tape recorded measuring data processing.]

gistrement. Il est alors connecté en application fréquence-tension conformément au schéma fourni dans la note d'application déjà citée. Dans cette application, le module de conversion est une boucle du premier ordre qui délivre une tension proportionnelle à la fréquence d'entrée. Le coefficient de proportionnalité de l'ensemble est égal au produit du facteur d'échelle (adapté aux conditions de l'expérience) du convertisseur par le gain de l'amplificateur de sortie.

- Les signaux de synchronisation mémorisés sur la deuxième piste sont traités afin d'être mis en conformité avec la logique de l'ordinateur utilisé.

\section{Performances de l'ensemble.}

Des essais préalables ont été effectués pour vérifier les caractéristiques techniques de l'enregistreur utilisé, en particulier sa fidélité dans la reproduction des fréquences enregistrées, dans la gamme $50 \mathrm{~Hz}$, $15 \mathrm{kHz}$.

Pour qualifier la chaîne complète, il a ensuite été procédé à des tests de réponse aux transitoires pour les deux types de signaux d'entrée possibles.

Un signal en créneaux de basse fréquence est, pour cela, envoyé sur la partie interfaces $U / F-F / U$ ou $F \times N-F / U$ de la chaîne. Il est comparé à sa sortie au signal d'entrée après acquisition et traitement sur deux voies ordinateur.

4.1 Cas des SignauX de mesure analogiQues. Le schéma synoptique utilisé est celui de la figure 4 . Les résultats obtenus sont illustrés par les diagrammes de la figure 5. La réponse est dans ce cas du premier ordre. Le temps de réponse à $5 \%$ près [5] est de $130 \mathrm{~ms}$, ce qui correspond à une constante de temps de l'ordre de $45 \mathrm{~ms}$.

4.2 CAS DES SIGNAUX LOGIQUES. - Le schéma synoptique utilisé est celui de la figure 6 .

Afin de faciliter l'exploitation des informations de 


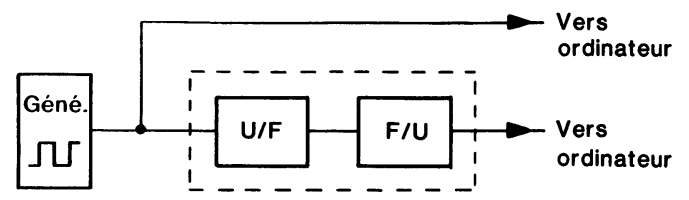

Fig. 4. - Test de réponse à un signal d'entrée analogique : schéma synoptique.

[Response test to an input analog signal : block diagram.]

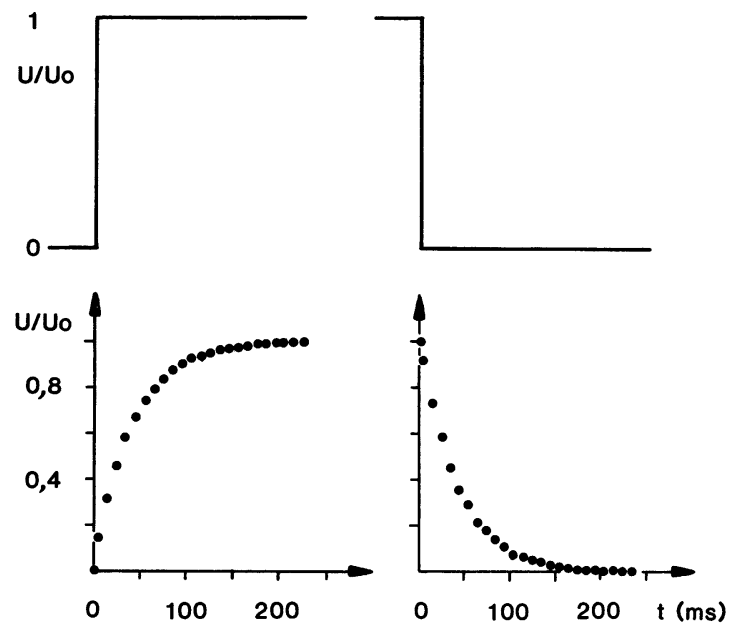

Fig. 5. - Interface analogique : réponse en transitoire. $U_{0}$ : tension maximum.

[Analog interface : transient response. $U_{0}$ maximum voltage.]

test par l'ordinateur, il est procédé à une conversion tension-fréquence avant d'attaquer le montage à tester. Ce temps de conversion est en fait de peu d'importance dans ce test face au temps de réponse de la suite de la chaîne. La figure 7 illustre les résultats obtenus. Le temps de réponse au bout duquel le régime définitif est atteint à $\pm 5 \%$ et ne s'en écarte pas de plus de $5 \%$ [6] est de $270 \mathrm{~ms}$ pour la partie ascendante du créneau et de $360 \mathrm{~ms}$ pour la partie descendante.

\section{Exemple d'utilisation.}

Nous décrivons brièvement, comme exemple d'utilisation, la mise en œuvre d'un ensemble destiné à l'acquisition des mesures du vent et des paramètres de fonctionnement d'une éolienne devant servir à alimenter le phare de l'Ile Amédée en Nouvelle Calédonie et qui sera mise en place prochainement par le Service Technique des Phares et Balises. L'objectif de ces mesures est d'évaluer l'effet des rafales de vent sur l'énergie produite par la machine. Les essais de qualification de la chaîne décrite ici, ont été conduits avec la collaboration de la subdivision du STPB d'Aix-en-Provence, sur le site de Vitrolles,

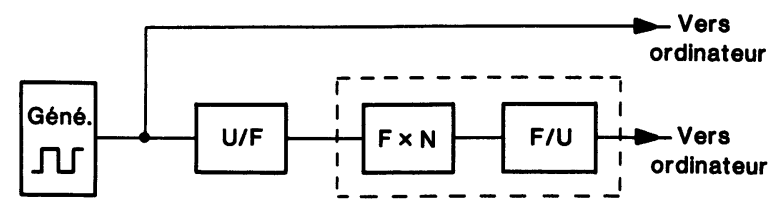

Fig. 6. - Test de réponse à un signal d'entrée logique : schéma synoptique.

[Response test to an input logic signal : block diagram.]
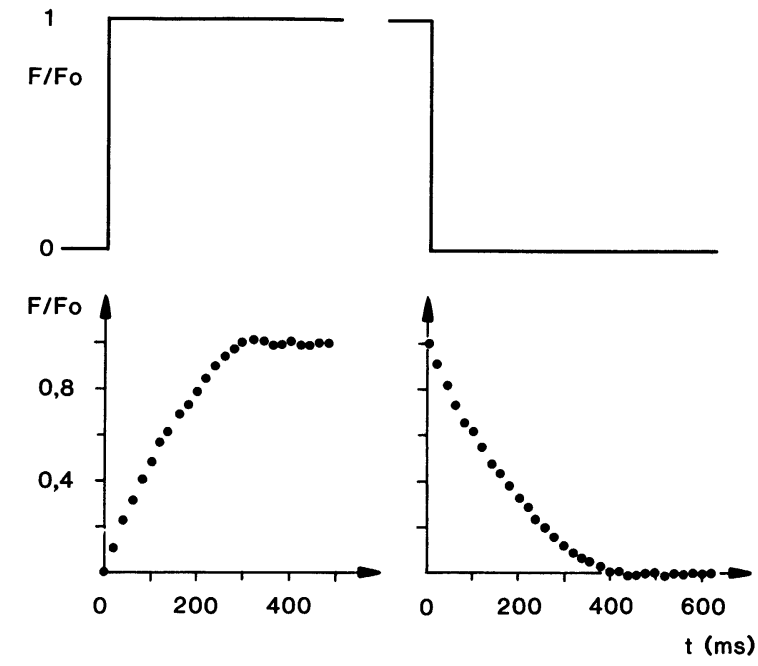

Fig. 7. - Interface logique : réponse en transitoire. $F_{0}$ : fréquence maximum.

[Logic interface : transient response. $F_{0}:$ maximum frequency.]

sur l'une de leurs machines de $1100 \mathrm{~W}$ de puissance nominale.

La chaîne d'acquisition comporte, dans l'exemple décrit ici, sept voies de mesure. Cinq d'entre elles traitent des signaux analogiques : ceux issus de potentiomètres donnant l'orientation du vent $\psi$ et de l'aérogénérateur $\varphi_{\mathrm{e}}$ le calage des deux pales $\theta$ et $\theta_{\mathrm{p}}$, et celui provenant du convertisseur puissancetension $P$. Les deux autres traitent des signaux logiques issus de cellules opto-électroniques fournissant la vitesse de rotation de l'anémomètre à coupelle (donc la vitesse du vent $V$ ) et celle de l'hélice $\Omega$.

Les résultats prétraités sont représentés sur la figure 8. Les enregistrements réalisés sur le site de Vitrolles ont été relus par l'ordinateur NORD 100 du C.E.A.T. équipé d'un ensemble d'acquisition CAMAC. On a réalisé, pour chaque paramètre, un échantillonnage chaque $1 / 10^{\mathrm{e}}$ de seconde pendant 150 secondes. Les mesures peuvent alors être traitées numériquement (analyse spectrale, corrélations, etc.) afin de fournir les informations souhaitées sur le fonctionnement de l'éolienne. 


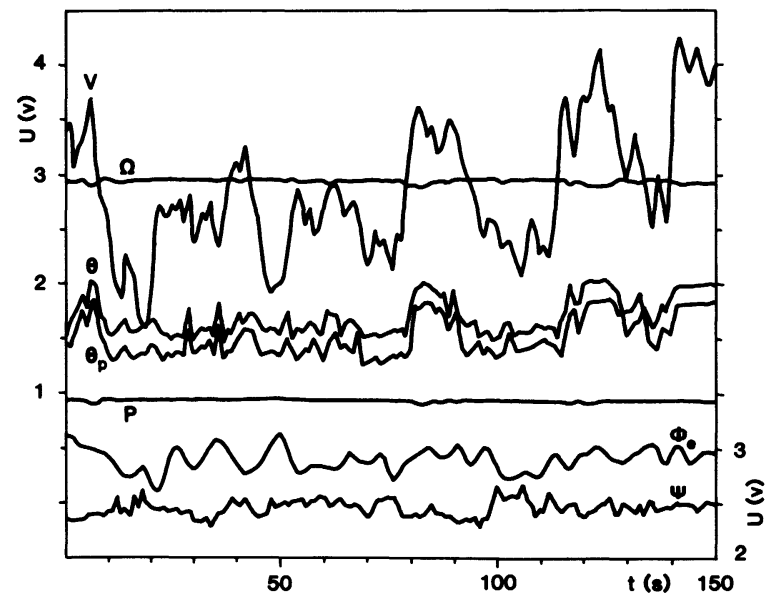

Fig. 8. - Exemple de résultats obtenus après traitement.

[Example of results obtained after processing.]

\section{Comparaison avec d'autres matériels.}

La solution adoptée ici a été retenue en raison de son faible coût (moins de $7500 \mathrm{~F}$ en janvier 84 pour 7 voies de mesures complètes) en comparaison du matériel existant sur le marché.

- Une chaîne d'acquisition de type HewlettPackard, Datel... L'information est alors stockée sous forme numérique. Le coût de ce type de matériel (janvier 84) est compris entre 40000 et $50000 \mathrm{~F}$ pour 8 voies dans le cadre de l'utilisation envisagée ici (acquisition sur une durée minimale d'une trentaine de minutes avec au moins 1 mesure par voie toutes les $100 \mathrm{~ms}$ ).

- Enregistreur magnétique continu dont le prix pour 8 voies en janvier 1984 est supérieur à $250000 \mathrm{~F}$.

- Un multiplexeur analogique suivi d'un seul enregistreur magnétique : en fait cette solution n'est envisageable que pour des fréquences d'échantillonnage faibles : en effet la méthode de conversion des convertisseurs $U / F$ est en général, assez lente, par conséquent la fréquence maxi d'échantillonnage étant égale à $1 / t \times n$ ( $t$ est le temps de conversion du convertisseur et $n$ le nombre de voies d'entrée sur le multiplexeur), un nombre de voies supérieur à 10 conduit déjà à une fréquence d'échantillonnage maximale inférieure à $1 \mathrm{~Hz}$. Ce sont donc les performances du convertisseur plus que celles de l'enregistreur qui créent une limitation. On souhaite en effet une mesure par voie tous les $1 / 10^{\mathrm{e}} \mathrm{s}$.

L'usage d'un seul enregistreur nécessite donc l'emploi d'autres modes de conversion et d'un autre type de modulation. On retrouve la solution de la chaîne d'acquisition décrite plus haut.

Ainsi, même si le coût "laboratoire" de $7500 \mathrm{~F}$ doit être réévalué dans le cadre d'une production industrielle, la chaîne proposée ici restera très compétitive et une petite société Poitevine est intéressée par la fabrication de ce matériel qui pourrait être commercialisé par la société DELTALAB avec laquelle des contacts ont été pris.

\section{Conclusion.}

La chaîne d'acquisition de mesures réalisée permet, pour une consommation par voie n'excédant pas $130 \mathrm{~mA}$, de traiter en régime permanent avec une précision inférieure à $0,8 \%$ (soit $0,3 \%$ pour le convertisseur $\mathrm{AD}$ et les composants externes $\mathrm{R}$ et $\mathrm{C}$ et $0,5 \%$ pour les taux de pleurage et de scintillement des enregistreurs) des tensions comprises entre $50 \mathrm{mV}$ et $10 \mathrm{~V}$ et des fréquences allant de $8 \mathrm{~Hz}$ à $400 \mathrm{~Hz}$. En régime transitoire, on peut traiter des variations de tension de $4 \mathrm{~V}$ par $1 / 10^{\mathrm{e}}$ de seconde et des variations de fréquence pouvant atteindre $80 \mathrm{~Hz}$ par $1 / 10^{\mathrm{e}}$ de seconde.

Le procédé classique de modulation de fréquence utilisé dans les enregistreurs analogiques du commerce est remplacé ici par une méthode de conversion plus simple à mettre en œuvre. L'ensemble réalisé ainsi, avec des composants courants donc peu coûteux et de bonne fiabilité, léger et de faible consommation offre une solution originale et économique au problème du traitement in situ des phénomènes à fréquence modérément variable.

\section{Remerciements.}

L'auteur remercie pour ses conseils Mr. R. Leblanc, du Groupe Energie Eolienne du C.E.A.T., ainsi que Mr. P. Ambaud, du Service Technique des Phares et Balises d'Aix-en-Provence qui a dirigé la construction et organisé les essais à Vitrolles du prototype d'éolienne servant de support à la qualification de la chaîne décrite ici. Sa reconnaissance va aussi à Mr. G. Bals, chercheur à l'ENSMA de Poitiers, pour son aide lors de ces essais.

\section{Bibliographie}

[1] Fontenay, R., Convertisseurs (Ed. Radio, Paris) 1979, p. 94.

[2] TRAn TIEn LANG, Electronique des systèmes de mesures (Collection Mesures Physiques, Ed. Masson, Paris) 1983, p. 157.

[3] Oенміснеn, Signaux et circuits électroniques (Ed. Radio, Paris) 1979, p. 225.

[4] Damaye, R., Les oscillateurs (Ed. Techniques et Scien- tifiques Françaises, Collection Scientifique Contemporaine, Paris) p. 287.

[5] Asch, G. et al., Les capteurs en instrumentation industrielle (Ed. Dunod, Paris) 1983, p. 37.

[6] Gille, J. C., Decaulne, P., Pelegrin, M., Théorie et calcul des asservissements (Ed. Dunod, Paris) 1958 , p. 60. 